\title{
Carbapenem-Resistant Klebsiella pneumoniae Osteomyelitis Treated with Ceftazidime-Avibactam in an Infant: A Case Report
}

\author{
Zejuan Ji' \\ Keming Sun' \\ Zhenwei $\mathrm{Li}^{1}$ \\ Weyland Cheng $\left(^{1,2}\right.$ \\ Junwen Yang ${ }^{3}$ \\ 'Department of Orthopaedic Surgery, \\ Children's Hospital Affiliated to \\ Zhengzhou University, Henan Children's \\ Hospital, Zhengzhou Children's Hospital, \\ Zhengzhou, Henan, People's Republic of \\ China; ${ }^{2}$ Henan Provincial Key Laboratory \\ of Children's Genetics and Metabolic \\ Diseases, Children's Hospital Affiliated to \\ Zhengzhou University, Henan Children's \\ Hospital, Zhengzhou Children's Hospital, \\ Zhengzhou, Henan, People's Republic of \\ China; ${ }^{3}$ Microbiology Laboratory, \\ Children's Hospital Affiliated to \\ Zhengzhou University, Henan Children's \\ Hospital, Zhengzhou Children's Hospital, \\ Zhengzhou, Henan, People's Republic of \\ China
}

Correspondence: Zejuan Ji; Weyland Cheng

Tel +86 I8538557605; +86 I8502758200 Email Jizejuan_Cristina@126.com; wey_ c@hotmail.com

\begin{abstract}
Increasing cases of carbapenem-resistant Klebsiella pneumoniae (CR-KP) infections have been observed globally where multi-drug resistance to CR-KP can make the infection difficult to treat. In recent years, the $\beta$-lactam/ $\beta$-lactamase inhibitor, ceftazidimeavibactam (CAZ-AVI), has been developed to treat complicated urinary tract infections and complicated intra-abdominal infections. CAZ-AVI is approved for children over 3-month old but has yet to be investigated for cases of osteomyelitis. Only three case reports exist in literature on the use of CAZ-AVI for CR-KP osteomyelitis in adults. In this report, we present an infant with primary hematogenous osteomyelitis and septic arthritis in the right shoulder following surgical treatment for a heart murmur. Bacterial isolation revealed a strain of CR-KP, which was successfully treated with CAZ-AVI after initial administration of imipenem-based treatments.
\end{abstract}

Keywords: carbapenem-resistant Enterobacteriaceae, multi-drug resistant, $\beta$-lactam $/ \beta$ lactamase inhibitor, antibiotic, susceptibility test

\section{Introduction}

Osteomyelitis is an infection of the bone typically caused by Staphylococcus aureus in children, of which a portion of these cases consist of methicillin-resistant S. aureus. ${ }^{1}$ Carbapenem-resistant Klebsiella pneumoniae (CR-KP) is a less common source of infection, but incidences of CR-KP have been increasing in recent years, in part due to the widespread use of carbapenem. ${ }^{2}$ CR-KP is a multi-drug resistant Gram-negative bacillus that produces enzymes such as carbapenemases, which degrades carbapenem. ${ }^{3}$ The mortality rate for patients with CR-KP can range from $22 \%$ to $45 \%$ with an even higher mortality rate for patients that are coinfected or patients who have comorbidities. ${ }^{4}$

$\beta$-lactam/ $\beta$-lactamase inhibitors such as ceftazidime-avibactam (CAZ-AVI) and aztreonam-avibactam (ATM-AVI) are relatively new drugs that can be used to treat carbapenem-resistant Enterobacteriaceae (CRE). ${ }^{3}$ CAZ-AVI has been shown to have potent activity in vitro against non-metallo- $\beta$-lactamase producing CREs. However, KPC-2-expressing CR-KP isolates may show resistance. Alternatively, ATM-AVI was shown to be potent for all CRE types, but has yet to be FDA approved for clinical use. 
Yet, no clinical trials have been conducted on the use of CAZ-AVI for severe osteomyelitis. Three case reports exist in literature on the use of CAZ-AVI for managing CR-KPinduced osteomyelitis in adults. ${ }^{5-7}$ In this article, we present an infant with primary hematogenous osteomyelitis and septic arthritis caused by CR-KP and treated with CAZ-AVI after initial resistance to an imipenem-based antibiotic therapy.

\section{Case Presentation}

On November 13, 2020, a one month and 15-day old male infant was admitted to the hospital due to a heart murmur that persisted over 7 days. Upon admission, the infant showed good mental response, even breathing, no congestion in the pharynx, clear breathing sounds in both lungs, and no rales. Heart rate was 136 beats/min and SMIII/6 grade murmurs could be heard between the 3 and 4 intercostals on the left edge of the sternum, which was jet-like, widespread and P2 hyperactive. A surgical procedure was conducted on November 16, 2020 under general anesthesia and hypothermic cardiopulmonary bypass. Ventricular septal defect repair, patent foramen ovale repair, arterial duct ligation and open tricuspid valve angioplasty were conducted.

One-month post-operation, MRI assessment revealed incidence of septic arthritis in the right shoulder and osteomyelitis (Figure 1), to which the child was transferred to the Department of Orthopaedic Surgery on December 20, 2020. The infant's body temperature was $38^{\circ} \mathrm{C}$. Routine blood examination showed white blood cell (WBC) count $12,510 / \mu \mathrm{L}(>9150 / \mu \mathrm{L})$, erythrocyte sedimentation rate (ESR) $47 \mathrm{~mm} / \mathrm{h}(>15 \mathrm{~mm} / \mathrm{h})$, plateletcrit (PCT) $0.202 \mathrm{ng} / \mathrm{mL}(>0.05 \mathrm{ng} / \mathrm{mL})$, IL-6 $41.63 \mathrm{pg} / \mathrm{mL}(>7$ $\mathrm{pg} / \mathrm{mL}$ ), alanine aminotransferase (ALT) $12.0 \mathrm{U} / \mathrm{L}$, aspartate aminotransferase (AST) $33.1 \mathrm{U} / \mathrm{L}$, alkaline phosphatase (ALP) $277.4 \mathrm{U} / \mathrm{L}$, total bilirubin $7.8 \mu \mathrm{mol} / \mathrm{L}$ and direct bilirubin $4.3 \mu \mathrm{mol} / \mathrm{L}$. The infant was initiated on antibiotic therapy of imipenem/cilastatin (60 $\mathrm{mg} \mathrm{q} 6 \mathrm{~h}$ ).

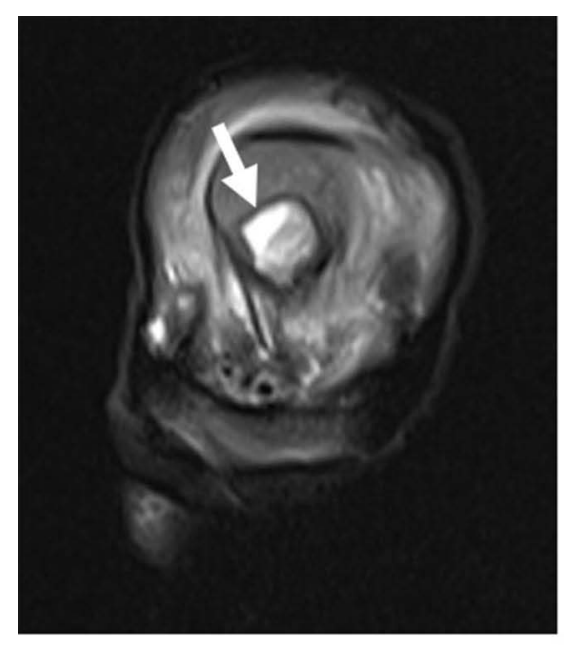

A

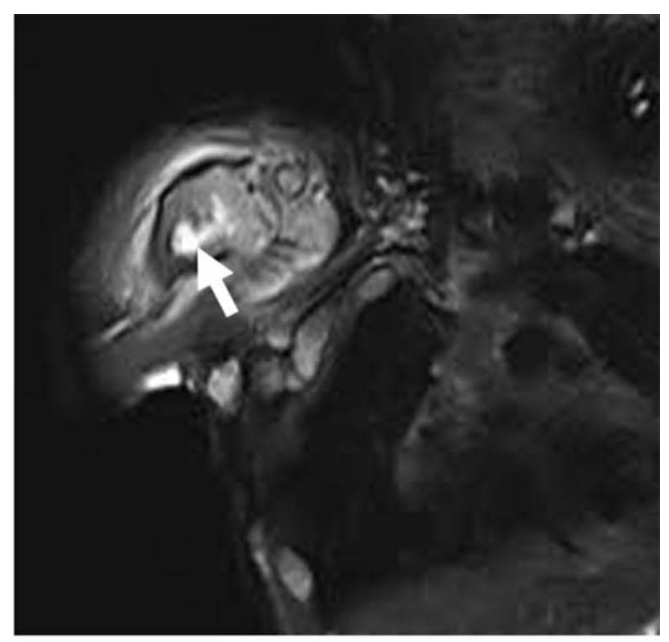

B

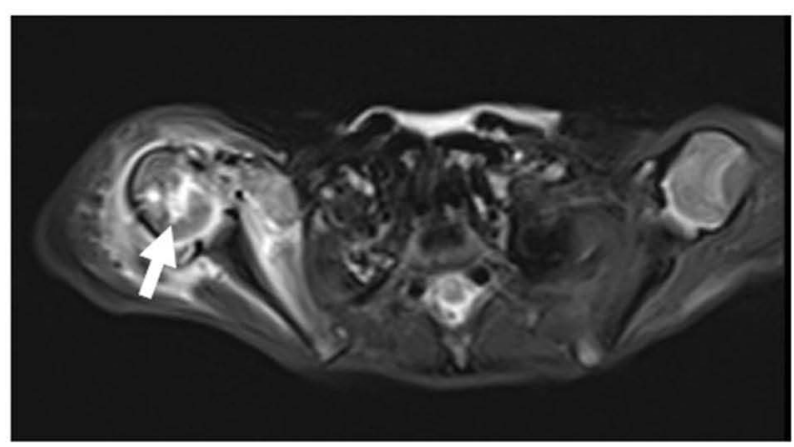

C

Figure I MRI showing bone marrow edema (white arrow) in the right shoulder in the (A) sagittal view, (B) coronal view, and (C) axial view. 
On December 21, 2020, right humerus fenestration along with the incision and drainage of the right shoulder joint was conducted. A large amount of pus was noted in the joint during operation. Blood and bone marrow fluid culture showed a bacterial infection of $K$. pneumoniae. Five days post-operation, pus was observed in the drainage tube. Upon recommendation from the Department of Pharmacy, imipenem/cilastatin (60 mg q6h) and fosfomycin (440 mg q8h) was administered for three days. After three days of medication, infection indices were still elevated upon reexamination and the drainage tube was still purulent.

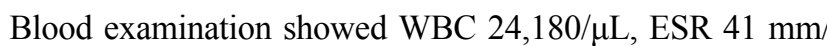
$\mathrm{hr}$, PCT $0.069 \mathrm{ng} / \mathrm{mL}$ and IL-6 $21.68 \mathrm{pg} / \mathrm{mL}$. Drug susceptibility testing of the isolated CR-KP strain showed susceptibility to trimethoprim/sulfamethoxazole (TMP-SMX), colistin (polymyxin E), CAZ-AVI and tigecycline (Table 1).

A second surgery was conducted on December 29, 2020 where a large amount of pus and damage to the humeral head was noted during operation. Surgical debridement and drainage of the right humerus and right shoulder joint lesions was performed. After approval from the drug ethics committee of the hospital, CAZ-AVI was administered (200 $\mathrm{mg} \mathrm{q} 8 \mathrm{~h}$ ) post-operation on December 29, 2020. Within 48 hours of administration, fluid in the drainage tube was noted to be clear and no pus was observed. The infant's body temperature, mental reaction and diet were normal. No adverse effects were observed during the administration of CAZ-AVI.

A third operation was conducted on January 12, 2021, for the debridement and drainage of the right humerus and right shoulder joint lesions. Blood examination prior to the surgery showed WBC $12,220 / \mu \mathrm{L}$, ESR $15 \mathrm{~mm} / \mathrm{h}$, PCT $0.067 \mathrm{ng} / \mathrm{mL}$ and IL-6 $14.21 \mathrm{pg} / \mathrm{mL}$. During the operation, no pus was observed in the joint, there was fresh tissue growth, and there was no further damage to the humeral head. Upon noting the eradication of infection, administration of CAZ-AVI was discontinued post-surgery to which meropenem (200 mg q8h) was maintained. On January 19, 2021, laboratory results showed WBC $12,690 / \mu \mathrm{L}$, РCT $0.043 \mathrm{ng} / \mathrm{mL}$, IL- $6<1.50 \mathrm{pg} / \mathrm{mL}$, ESR $9 \mathrm{~mm} / \mathrm{h}$, ALT $17.4 \mathrm{U} / \mathrm{L}$, AST $30.9 \mathrm{U} / \mathrm{L}$, total bilirubin $6.4 \mu \mathrm{mol} / \mathrm{L}$, and direct bilirubin $1.6 \mu \mathrm{mol} / \mathrm{L}$.

\section{Discussion}

In recent studies, carbapenem and non-beta-lactam betalactamase inhibitors, such as imipenem-relebactam, have been found to be effective against most strains of CR-KP and clinical trials show favorable responses to imipenem/ cilastatin/relebactam. ${ }^{8,9}$ However, the in vivo study of imipenem-relebactam against CREs still showed poor activity against 23 metallo-carbapenemase producers out of 660 isolates. $^{9}$ An analysis of CR-KP strains in Chongqing, China, found that among carbapenems, $100 \%$ of the CR-KP isolates were resistant to ertapenem, $37.2 \%$ were resistant to imipenem, $30.8 \%$ were resistant to meropenem ${ }^{2}$ Resistance to cephalosporins were relatively high among $52.5 \%, 75.6 \%$ and $78.2 \%$ isolates against cefepime, CAZ and ceftriaxone, respectively. Fluoroquinolone resistance was tested to be $59 \%$ and $43.6 \%$ against ciprofloxacin and levofloxacin, respectively.

Table I Susceptibility of Antibiotics to the CR-KP Isolate

\begin{tabular}{|c|c|c|c|c|c|}
\hline Antibiotic & $\mathbf{R} / \mathbf{S}$ & MIC mg/L & Antibiotic & $\mathbf{R} / \mathbf{S}$ & MIC mg/L \\
\hline Amikacin & $\mathrm{R}$ & $>32$ & Colistin (polymyxin E) & $S$ & $\leq 1$ \\
\hline Amoxicillin/Clavulanic & $\mathrm{R}$ & $>32 / 16$ & Gentamicin & $\mathrm{R}$ & $>8$ \\
\hline Ampicillin/Sulbactam & $\mathrm{R}$ & $>16 / 8$ & Imipenem & $\mathrm{R}$ & $>8$ \\
\hline Aztreonam & $\mathrm{R}$ & $>32$ & Meropenem & $\mathrm{R}$ & $>8$ \\
\hline Cefazolin & $\mathrm{R}$ & $>16$ & Minocycline & I & 8 \\
\hline Cefepime & $\mathrm{R}$ & $>16$ & Moxifloxacin & $\mathrm{R}$ & $>2$ \\
\hline Cefoperazone/Sulbactam & $\mathrm{R}$ & $>32 / 8$ & Norfloxacin & $\mathrm{R}$ & $>8$ \\
\hline Ceftazidime & $\mathrm{R}$ & $>32$ & Piperacillin/tazobactam & $\mathrm{R}$ & $>64 / 4$ \\
\hline Ceftazidime/avibactam & $S$ & 24 & Tetracycline & $\mathrm{R}$ & $>8$ \\
\hline Ceftriaxone & $\mathrm{R}$ & $>32$ & Tigecycline & S & 2 \\
\hline Cefuroxime & $\mathrm{R}$ & $>16$ & Tobramycin & $\mathrm{R}$ & $>8$ \\
\hline Chloramphenicol & I & 16 & Trimethoprim/sulfamethoxazole & $S$ & $\leq 1 / 19$ \\
\hline Ciprofloxacin & $\mathrm{R}$ & $>4$ & & & \\
\hline
\end{tabular}

Abbreviations: ALP, alkaline phosphatase; ALT, alanine aminotransferase; AST, aspartate aminotransferase; ATM-AVI, aztreonam-avibactam; CAZ-AVI, ceftazidimeavibactam; CR-KP, Carbapenem-resistant Klebsiella pneumoniae; ESR, erythrocyte sedimentation rate; PCT, plateletcrit; TMP-SMX, trimethoprim/sulfamethoxazole; WBC, white blood cell. 
Lastly, resistance of isolates to aminoglycoside was at $47.4 \%$ and $24.4 \%$ in gentamicin and tobramycin, respectively.

Regarding the administration of CAZ-AVI in cases of bacterial osteomyelitis, Cani et al (2018) reported the use of CAZ-AVI in a case of CR-KP vertebral osteomyelitis in a 60-year-old woman who underwent corrective spinal surgery. ${ }^{5}$ The authors administered $2.5 \mathrm{~g}$ IV of CAZ-AVI every 8 hours, initially combined with amikacin. Their time-kill studies indicated that CAZ-AVI only had synergy with meropenem whereas amikacin, polymyxin B and tigecycline were not synergetic; thus, amikacin was discontinued after 13 days. A six-week therapy resolved the infection.

De León-Borrás et al (2018) reported a case of a 36year-old man who had refractory CR-KP bacteremia, vertebral diskitis and osteomyelitis with pre-vertebral abscess and bilateral psoas pyomyositis. ${ }^{6}$ Initial treatment of polymyxin B, carbapenems, and amikacin for six weeks was found to be ineffective. The patient was switched to CAZAVI treatment for another six weeks to which the infection was completely resolved.

A third case, reported by Schimmenti et al (2018), presented a 26-year-old male with CR-KP osteomyelitis of the right distal femur from a prosthetic joint infection. ${ }^{7}$ Implants of the patient was removed and a fistulectomy of the right femur was conducted. Bacterial isolates from the biopsy and sonification of the prosthetic plate showed the same strain of CR-KP. The patient was administered colistin-fosfomycin and trimethoprim-sulfamethoxazole, which was later changed to colistin, fosfomycin and tigecycline. After additional surgical resection and upon poor tolerance towards the treatment, CAZ-AVI at a dose of $2.5 \mathrm{~g}$ three times a day for two weeks was administered following susceptibility testing, which successfully treated the infection.

In this case presentation, we found that at two weeks of administration, CAZ-AVI (50 mg/kg q8h) was effective and safe in treating primary CR-KP osteomyelitis in a male infant whereas the administration of imipenem/ cilastatin and imipenem/cilastatin/fosfomycin was clinically ineffective. The dosage of $50 \mathrm{mg} / \mathrm{kg}$ was chosen based on manufacturer guidelines for infants 3 months to less than 6 months of age. At the time of CAZ-AVI administration, the infant just reached three months old. Susceptibility testing conducted after initial drug administration determined that the isolated CR-KP strain was resistant to both imipenem and fosfomycin.
The isolate was found to be susceptible to colistin, TMP-SMX and tigecycline, but these antibiotics were not selected as treatment options. Tigecycline can be unfavorable due to adverse effects, increased mortality rates and increased noncure rates as shown in a meta-analysis based on noninferiority trials. ${ }^{10}$ Polymyxin $\mathrm{B}$ can reduce mortality rates of CR-KP bloodstream infections in adults. ${ }^{11}$ However, pharmacokinetic and pharmacodynamic data on the use of polymyxin in neonates and children are limited and adverse events can include nephrotoxicity and neurotoxicity, thus causing concern for safety. ${ }^{12}$ TMPSMX is a potential option for acute osteomyelitis infections and suitable for children two months of age and older. TMP-SMX has several US FDA-approved indications, which do not include osteomyelitis. In one study, a trial consisting of 20 children with acute osteomyelitis were successfully treated using TMP-SMX for 26-59 days with mild adverse effects. ${ }^{13}$ However, controversy exists in its use due to the release of thymidine from damaged tissue and bacteria, which can interfere with the bactericidal activity of antifolates such as TMP-SMX. ${ }^{14}$ Thus, in vitro susceptibility testing may not be representative of in vivo clinical efficacy.

CAZ-AVI, on the other hand, is US FDA approved for treating children over 3 months old who have complicated urinary tract infections and complicated intra-abdominal infections (treated in combination with metronidazole). In the case series by Iosifidis et al (2019), eight neonates or children $<5$ years of age were successfully treated for infections caused by extensively drug-resistant or pan drug-resistant KP using 9 courses of CAZ-AVI at a $62.5 \mathrm{mg} / \mathrm{kg}$ q $8 \mathrm{~h} .{ }^{15}$ No significant adverse events were reported in their study. Thus, CAZ-AVI can be a viable and safe option for infants with CR-KP related infections. However, early susceptibility testing is still essential to determine the proper treatment as incidences of CAZAVI resistant CR-KP have been previously reported. ${ }^{16}$

\section{Conclusion}

This report is the first published case regarding the use of CAZ-AVI for CR-KP osteomyelitis in a child. CREinduced osteomyelitis has yet to be listed as an approved indication for the administration of CAZ-AVI and optimal treatment dosages and durations for osteomyelitis have yet to be investigated. In concordance with previous case reports in adults, we recommend clinical studies towards the development of CAZ-AVI for use in CR-KP osteomyelitis cases among children and adults. Early susceptibility 
testing after bacterial isolation in cases of CR-KP osteomyelitis should be conducted to reach optimal treatment decisions and to prevent progression to osteonecrosis.

\section{Ethics Approval and Informed Consent}

The drug ethics committee of Children's Hospital Affiliated to Zhengzhou University (Henan Children's Hospital) approved the use of ceftazidime-avibactam. Permission to publish was obtained from the Ethics Committee of Henan Children's Hospital (No. 2021$\mathrm{K}-055)$. Informed consent was obtained from the parent.

\section{Consent for Publication}

Informed consent to publish was obtained from the parent.

\section{Disclosure}

The authors report no conflicts of interest in this work.

\section{References}

1. McNeil JC. Acute hematogenous osteomyelitis in children: clinical presentation and management. Infect Drug Resist. 2020;13:4459-4473. doi:10.2147/IDR.S257517

2. Yan J, Pu S, Jia X, et al. Multidrug resistance mechanisms of carbapenem resistant Klebsiella pneumoniae strains isolated in Chongqing, China. Ann Lab Med. 2017;37(5):398-407. doi:10.3343/ alm.2017.37.5.398

3. Wei J, Zou C, Wang D, Huang A, Niu S. Genetic diversity and in vitro activity of ceftazidime/avibactam and aztreonam/avibactam against imipenem-resistant Enterobacteriaceae isolates in Southwest China: a single-centre study. J Glob Antimicrob Resist. 2020;22:448-451. doi:10.1016/j.jgar.2020.04.023

4. Munoz-Price LS, Poirel L, Bonomo RA, et al. Clinical epidemiology of the global expansion of Klebsiella pneumoniae carbapenemases. Lancet Infect Dis. 2013;13(9):785-796. doi:10.1016/S1473-3099(13)70190-7

5. Cani E, Moussavi F, Ocheretyaner E, Sharma R, Brown C, Eilertson B. Carbapenem-resistant Klebsiella pneumoniae vertebral osteomyelitis in a renal transplant recipient treated with ceftazidime-avibactam. Transpl Infect Dis. 2018;20(2):e12837. doi:10.1111/tid.12837
6. De Leon-borras R, Alvarez-Cardona J, Vidal JA, Guiot HM. Ceftazidime/avibactam for refractory bacteremia, vertebral diskitis/ osteomyelitis with pre-vertebral abscess and bilateral psoas pyomyositis secondary to Klebsiella Pneumoniae Carbapenemase-Producing Bacteria (KPC). P R Health Sci J. 2018;37(2):128-131.

7. Schimmenti A, Brunetti E, Seminari E, Mariani B, Cambieri P, Orsolini P. Prosthetic joint infection from carbapenemase-resistant Klebsiella pneumoniae successfully treated with ceftazidime-avibactam. Case Rep Infect Dis. 2018;2018:1854805.

8. Kohno S, Bando H, Yoneyama F, et al. The safety and efficacy of relebactam/imipenem/cilastatin in Japanese patients with complicated intra-abdominal infection or complicated urinary tract infection: a multicenter, open-label, noncomparative phase 3 study. $J$ Infect Chemother. 2021;27(2):262-270. doi:10.1016/j.jiac.2020.09.032

9. Yang TY, Hsieh YJ, Kao LT, et al. Activities of imipenem-relebactam combination against carbapenem-nonsusceptible Enterobacteriaceae in Taiwan. J Microbiol Immunol Infect. 2021. doi:10.1016/j. jmii.2021.02.001

10. Prasad P, Sun J, Danner RL, Natanson C. Excess deaths associated with tigecycline after approval based on noninferiority trials. Clin Infect Dis. 2012;54(12):1699-1709. doi:10.1093/cid/cis270

11. Liang Q, Huang M, Xu Z. Early use of polymyxin B reduces the mortality of carbapenem-resistant Klebsiella pneumoniae bloodstream infection. Braz J Infect Dis. 2019;23(1):60-65. doi:10.1016/ j.bjid.2018.12.004

12. Thomas R, Velaphi S, Ellis S, et al. The use of polymyxins to treat carbapenem resistant infections in neonates and children. Expert Opin Pharmacother. 2019;20(4):415-422. doi:10.1080/ 14656566.2018.1559817

13. Messina AF, Namtu K, Guild M, Dumois JA, Berman DM. Trimethoprim-sulfamethoxazole therapy for children with acute osteomyelitis. Pediatr Infect Dis J. 2011;30(12):1019-1021. doi:10.1097/INF.0b013e31822db658

14. Pezone I, Leone S. Role of Trimethoprim-sulfamethoxazole for treatment of acute osteomyelitis in children. Pediatr Infect Dis J. 2012;31 (6):660-661; author reply 661. doi:10.1097/INF.0b013e318255ff8d

15. Iosifidis E, Chorafa E, Agakidou E, et al. Use of Ceftazidimeavibactam for the treatment of extensively drug-resistant or pan drugresistant Klebsiella pneumoniae in neonates and children $<5$ years of age. Pediatr Infect Dis J. 2019;38(8):812-815.

16. Zhou J, Yang J, Hu F, Gao K, Sun J, Yang J. Clinical and molecular epidemiologic characteristics of ceftazidime/avibactam-resistant carbapenem-resistant Klebsiella pneumoniae in a neonatal intensive care unit in China. Infect Drug Resist. 2020;13:2571-2578. doi:10.2147/IDR.S256922
Infection and Drug Resistance

\section{Publish your work in this journal}

Infection and Drug Resistance is an international, peer-reviewed openaccess journal that focuses on the optimal treatment of infection (bacterial, fungal and viral) and the development and institution of preventive strategies to minimize the development and spread of resistance. The journal is specifically concerned with the epidemiology of antibiotic resistance and the mechanisms of resistance development and diffusion in both hospitals and the community. The manuscript management system is completely online and includes a very quick and fair peerreview system, which is all easy to use. Visit http://www.dovepress.com/ testimonials.php to read real quotes from published authors. 